\title{
Elevated Serum Uric Acid Levels Are Related to Cognitive Deterioration in an Elderly Japanese Population
}

\author{
Kazushi Suzukia, b Daisuke Koide ${ }^{c}$ Kurumi Fujii ${ }^{d}$ Tsutomu Yamazaki a, d \\ Shoji Tsuji $^{b}$ Atsushi Iwatab, e \\ ${ }^{a}$ Center for Epidemiology and Preventive Medicine, the University of Tokyo Hospital, Tokyo, \\ Japan; ${ }^{\mathrm{b}}$ Department of Neurology, the University of Tokyo, Tokyo, Japan; ' Department of \\ Clinical Epidemiology and Systems, the University of Tokyo, Tokyo, Japan; ${ }^{\mathrm{d} C l i n i c a l}$ Research \\ Support Center, the University of Tokyo Hospital, Tokyo, Japan; ' Japan Science and \\ Technology Agency, PRESTO, Tokyo, Japan
}

\section{Keywords}

Uric acid · Cognitive deterioration - Dementia - Mild cognitive impairment - Risk factor ·

White matter lesion

\begin{abstract}
Aims: The association between serum uric acid (UA) levels and cognitive function is controversial since UA can be a risk factor for cerebral ischemia as well as acting as a neuroprotective antioxidant. Methods: We conducted a cross-sectional analysis of 228 elderly participants and examined neuropsychological test results, clinical data as well as brain magnetic resonance imaging data. Patients: Overall, 64 participants were diagnosed with cognitive deterioration. To control for the effect of sex differences, 2 independent sets of single-variable and multivariate logistic regression analyses were performed with quartiles divided into non-sexspecific and sex-specific cutoff values for UA. Results: In non-sex-specific quartiles, the participants in the highest quartiles of UA levels were found to be at a significantly higher risk of cognitive deterioration than those in the lowest quartiles. In sex-specific quartiles, the highest quartile showed an increased risk of cognitive deterioration, and a greater than fourfold increase in the risk in the highest quartiles was confirmed using multivariate regression models. However, no significant association was observed between serum UA levels and the presence of white matter lesions. Conclusions: Elevated serum UA levels were independently associated with cognitive deterioration. UA might have unknown adverse effects on cognitive function, other than causing vascular pathology.


Suzuki et al.: Elevated Serum Uric Acid Levels Are Related to Cognitive Deterioration in an Elderly Japanese Population

\section{Introduction}

Uric acid (UA), which is the end product of purine metabolism, is an established risk factor for gout and urinary calculus. Recent epidemiological studies have shown a strong association between elevated serum UA levels and increased risk for myocardial infarction, stroke, and cardiovascular mortality [1-3]. The pathophysiological backgrounds of these positive associations are thought to be caused by vascular pathology, potentially due to UA. UA can stimulate vascular smooth muscle cell proliferation [4-6] and can also induce endothelial dysfunction by inhibiting nitric oxide production [7]. Elevated serum UA levels are also thought to be associated with systemic inflammation $[8,9]$. These pathologies also cause brain damage. Thus, elevated serum UA levels are regarded as a potent risk factor for the development of dementia. Supporting this hypothesis, some populationbased cross-sectional studies on community-dwelling older adults have demonstrated a positive association between the increase in serum UA levels and cognitive deterioration $[10,11]$.

On the other hand, some investigators believe that UA might have a beneficial effect on brain function. UA is a strong natural antioxidant, and potentially protects the cerebral tissues from oxidative stress caused by free radicals. In accordance with this hypothesis, higher serum levels of UA are reported to be associated with a significantly decreased risk of developing Parkinson disease [12]. Plasma UA levels were found to be reduced in patients with Alzheimer disease (AD) $[13,14]$; however, it is unknown whether this association is a reflection of the neuroprotective effects of UA or is the result of changes in the lifestyle of the patients with AD. Owing to these contradictory results, the association between serum UA levels and cognitive function remains controversial.

The aim of this study was to determine whether elevated serum UA levels could increase the risk for cognitive deterioration, independent of other health-related conditions and lifestyle factors. To this end, we conducted a cross-sectional analysis of neuropsychological test results, clinical data and brain magnetic resonance imaging (MRI) data, which were obtained from 228 individuals attending comprehensive medical checkups. We analyzed the brain MRI data of the participants in order to test for possible associations between asymptomatic white matter lesions (WML) and serum levels of UA. To adjust for the influence of sex differences on serum UA levels, we conducted an alternative analysis based on the sex-specific thresholds of serum UA levels.

\section{Materials and Methods}

\section{Participants}

Data for this study were obtained from voluntary participants in the "memory checkup," drawn from among individuals attending comprehensive medical checkups in the University of Tokyo Hospital between January 2009 and October 2011. This study was approved by the Institutional Review Board of the University of Tokyo Hospital. Participants were interviewed about their medical history, present health problems, and habits. They also underwent physical examinations, blood sampling, and 1.5-T brain MRI scans. Blood sampling and measurement of body weight were performed after $10 \mathrm{~h}$ of fasting. Systolic blood pressure was measured in a seated position after a 5-min rest using an automated blood pressure recorder, UDEX-Twin (ELK Corporation, Tokyo, Japan). MRI was performed using a 1.5-T Vantage scanner (Toshiba, Tokyo, Japan) and images were analyzed using the Advantage Workstation (GE Healthcare Japan, Tokyo, Japan). Periventricular hyperintensity and deep and subcortical white matter hyperintensities were evaluated by neurologists according to 
the criteria established by Fazekas et al. [15]. Patients with a periventricular hyperintensity grading of $>1$ or deep and subcortical white matter hyperintensities grading of $>1$ were defined as presenting with WML.

\section{Cognitive Tests and Diagnostic Criteria}

The participants underwent the Mini-Mental State Examination (MMSE), the Wechsler Memory Scale-Revised (WMS-R) Logical Memory (LM) story A immediate and delayed recall, and the Clock-Drawing Test (CDT). Each test was performed by neuropsychologists under the supervision of neurologists. A diagnosis of "cognitive deterioration" was established when the participant fulfilled at least 1 of the following 3 criteria: (a) MMSE score $<27$, (b) WMS-R LM story A delayed score lower than the cutoffs (education $\geq 16$ years: $>11 ; 10-15$ years: $>7$; 0-9 years: $>5$ ), and (c) having subjective complaints of forgetfulness and a CDT score $<4$. These criteria were minor modifications of the inclusion criteria for late amnestic mild cognitive impairment (MCI), as defined by the Japanese Alzheimer Disease Neuroimaging Initiative. The presence of subjective complaints of forgetfulness was determined by the answer to a single question: "Do you have any problems with your memory?"

\section{Laboratory Tests}

Laboratory tests were performed in the Department of Clinical Laboratory at the University of Tokyo Hospital, which is an accredited laboratory conforming to ISO 15189 standards. Serum UA, high-density lipoprotein (HDL)-cholesterol, low-density lipoprotein (LDL)-cholesterol, triglyceride, and creatinine levels were measured by spectrophotometry using a JCA-BM 8040 automated analyzer (Nihon Denshi, Tokyo, Japan). Fasting blood glucose was measured with the glucose oxidase-peroxidase method using a GA08II automated glucose analyzer (A\&T Corporation, Yokohama, Japan). The estimated glomerular filtration rate (eGFR) was calculated based on the following formula defined by Matsuo et al. [16]:

$$
\text { eGFR }\left(\mathrm{mL} / \mathrm{min} / 1.73 \mathrm{~m}^{2}\right)=194 \times \text { serum creatinine } \mathrm{s}^{-1.094} \times \operatorname{Age}^{-0.287}(\times 0.739, \text { if female }) .
$$

\section{Data Analysis}

A comparison between the 2 groups (cognitive deterioration/normal and male/female) was conducted using the Student $t$ test for numerical data and $\chi^{2}$ analysis for categorical data. The associations between serum UA levels and age, body weight, height, and body mass index (BMI) were examined using Pearson correlation coefficients. The associations between serum UA levels and sex, high/normal serum triglycerides, and high/normal fasting blood sugar levels were examined using $t$ tests. Owing to the skewed distributions, the data for serum triglyceride and fasting blood sugar levels were categorized into "high" and "normal/low" with cutoff values of $>149$ and $109 \mathrm{mg} / \mathrm{dL}$, respectively. These categorized data were used for association analyses with UA and logistic regression analyses, not for comparisons. The association between the quartiles of serum UA levels and the diagnosis of "cognitive deterioration" was analyzed using a single-variable logistic regression analysis. We also conducted a multivariate adjusted logistic regression analysis to adjust for the influence of other factors. The odds ratio (OR) was calculated to determine the risk for cognitive deterioration and WML with respect to serum UA levels; if the 95\% confidence interval (CI) did not include 1.0, the OR was considered to be statistically significant. Statistical analyses were performed using JMP (Version 9.0.0, SAS Institute Inc., Cary, NC, USA). 
Suzuki et al.: Elevated Serum Uric Acid Levels Are Related to Cognitive Deterioration in an Elderly Japanese Population

Table 1. Baseline characteristics of the 228 participants: comparisons between cognitive deterioration and normal groups, and between male and female individuals

\begin{tabular}{|c|c|c|c|c|c|c|}
\hline & $\begin{array}{l}\text { Cognitive } \\
\text { deterioration } \\
(n=64)\end{array}$ & $\begin{array}{l}\text { Normal } \\
(n=164)\end{array}$ & $p$ value & $\begin{array}{l}\text { Male } \\
(n=105)\end{array}$ & $\begin{array}{l}\text { Female } \\
(n=123)\end{array}$ & $p$ value \\
\hline Age, years & $68.0(10.4)$ & $62.9(9.7)$ & 0.0005 & $62.9(10.8)$ & $65.7(9.49)$ & 0.049 \\
\hline Sex (male) & $36(56.3)$ & $69(42.1)$ & 0.054 & - & - & - \\
\hline Education, years & $13.9(2.90)$ & $14.6(2.92)$ & 0.12 & $15.4(3.10)$ & $13.6(2.49)$ & $<0.0001$ \\
\hline Body weight, kg & $61.4(11.9)$ & $59.0(11.2)$ & 0.16 & $67.7(9.55)$ & $52.9(7.98)$ & $<0.0001$ \\
\hline Height, $\mathrm{cm}$ & $160.6(10.1)$ & $159.9(9.0)$ & 0.58 & $167.4(6.12)$ & $153.8(6.62)$ & $<0.0001$ \\
\hline BMI & $23.6(2.99)$ & $22.9(3.19)$ & 0.15 & $24.0(2.82)$ & $22.3(3.19)$ & $<0.0001$ \\
\hline \multicolumn{7}{|l|}{ Having subjective complaints } \\
\hline of forgetfulness & $45(70.3)$ & $119(72.6)$ & 0.73 & $71(68)$ & $93(76)$ & 0.18 \\
\hline Cognitive deterioration & - & - & - & $36(34)$ & $28(23)$ & 0.054 \\
\hline \multicolumn{7}{|l|}{ Neuropsychological test score } \\
\hline MMSE & $26.8(3.28)$ & $29.2(0.96)$ & $<0.0001$ & $28.4(2.26)$ & $28.6(2.14)$ & 0.54 \\
\hline WMS-R LM immediate & $9.08(3.94)$ & $15.71(4.03)$ & $<0.0001$ & $13.5(4.99)$ & $14.2(4.99)$ & 0.29 \\
\hline WMS-R LM delayed & $7.05(3.86)$ & $14.28(4.14)$ & $<0.0001$ & $11.8(5.19)$ & $12.7(5.21)$ & 0.2 \\
\hline $\mathrm{CDT}$ & $4.44(0.92)$ & $4.76(0.47)$ & 0.0006 & $4.65(0.66)$ & $4.69(0.63)$ & 0.61 \\
\hline Systolic blood pressure, $\mathrm{mm} \mathrm{Hg}$ & $124.9(17.2)$ & $120.0(16.1)$ & 0.044 & $122.7(15.93)$ & $120.2(17.03)$ & 0.25 \\
\hline LDL-cholesterol, mg/dL & $132.0(31.8)$ & $129.8(32.8)$ & 0.65 & $127.4(32.5)$ & $132.9(32.3)$ & 0.2 \\
\hline HDL-cholesterol, mg/dL & $67.0(19.8)$ & $68.1(16.3)$ & 0.67 & $60.0(14.7)$ & $74.4(16.6)$ & $<0.0001$ \\
\hline Triglyceride, mg/dL & $127.5(74.1)$ & $121.3(97.4)$ & 0.65 & $140.0(113.6)$ & $108.6(63.9)$ & 0.0093 \\
\hline Fasting blood sugar, $\mathrm{mg} / \mathrm{dL}$ & $107.7(23.0)$ & $102.9(19.6)$ & 0.11 & $108.0(21.9)$ & $101.0(19.1)$ & 0.0108 \\
\hline $\mathrm{eGFR}, \mathrm{mL} / \mathrm{min} / 1.73 \mathrm{~m}^{2}$ & $69.3(16.5)$ & 74.9 (12.8) & 0.0071 & $71.93(14.56)$ & $74.45(13.74)$ & 0.1805 \\
\hline Serum UA, mg/dL & $6.08(1.37)$ & $5.20(1.26)$ & $<0.0001$ & $6.23(1.27)$ & $4.78(1.01)$ & $<0.0001$ \\
\hline Presence of WML & $10(15.6)$ & $12(7.4)^{\mathrm{a}}$ & 0.058 & $9(8.7)^{a}$ & $13(10.6)$ & 0.63 \\
\hline \multicolumn{7}{|l|}{ Habits } \\
\hline Alcohol & $34(53)$ & $75(47)$ & 0.42 & $79(75)$ & $35(28)$ & $<0.0001$ \\
\hline Smoking & $27(33)$ & $37(26)$ & 0.26 & $63(60)$ & $20(16)$ & $<0.0001$ \\
\hline Exercise & $28(44)$ & $68(43)$ & 0.82 & $53(51)$ & $43(36)$ & 0.029 \\
\hline
\end{tabular}

\section{Results}

Between January 2009 and October 2011, 239 participants voluntarily underwent comprehensive medical checkups, including neuropsychological tests and brain MRI scans. Eleven participants were excluded from subsequent analyses as they regularly took antihyperuricemic agents. Among the remaining 228 participants, 28, 46, and 5 cases came under the cutoff values in the MMSE, WMS-R LM, and CDT tests, respectively. Ultimately, 64 individuals (36 men, 28 women) were diagnosed with cognitive deterioration (Table 1). The cognitive deterioration group and the normal group significantly differed in terms of age $\left(t_{226}=-3.53, p=0.0005\right)$, MMSE score $\left(t_{226}=8.45, p<0.0001\right)$, LM immediate score $\left(t_{226}=\right.$ $11.2, p<0.0001)$, LM late score $\left(t_{226}=12.1, p<0.0001\right)$, CDT score $\left(t_{226}=3.50, p=0.0006\right)$, systolic blood pressure $\left(t_{226}=2.03, p=0.044\right)$, eGFR $\left(t_{226}=-2.72, p=0.0071\right)$, and serum UA levels $\left(t_{226}=4.62, p<0.0001\right)$. However, these groups did not differ in terms of sex $\left(\chi^{2}(1, n=228)=3.72, p=0.0536\right)$, education $\left(t_{226}=1.53, p=0.128\right)$, body weight $\left(t_{226}=-1.39\right.$, $p=0.165)$, height $\left(t_{226}=-0.544, p=0.587\right)$, BMI $\left(t_{226}=-1.44, p=0.150\right)$, presence of WML $\left(\chi^{2}{ }_{(1, n=228)}=3.585, p=0.058\right)$, or presence of subjective complaints of forgetfulness $\left(\chi^{2}(1, n=228)=0.115, p=0.734\right)$. 
Suzuki et al.: Elevated Serum Uric Acid Levels Are Related to Cognitive Deterioration in an Elderly Japanese Population

Table 2. Crude ORs and multivariate-adjusted ORs for cognitive deterioration and for WML

\begin{tabular}{|c|c|c|c|c|c|c|c|}
\hline & \multirow[t]{2}{*}{ Group 1} & \multicolumn{2}{|l|}{ Group 2} & \multicolumn{2}{|l|}{ Group 3} & \multicolumn{2}{|l|}{ Group 4} \\
\hline & & OR $(95 \% \mathrm{CI})$ & $p$ value & OR $(95 \% \mathrm{CI})$ & $\mathrm{p}$ value & OR (95\% CI) & $p$ value \\
\hline \multicolumn{8}{|c|}{ Cognitive deterioration: non-sex-specific serum UA quartiles } \\
\hline \multicolumn{8}{|l|}{ Multivariate analyses } \\
\hline Model 1 & 1 & $1.77(0.68-4.81)$ & 0.24 & $2.20(0.79-6.33)$ & 0.13 & $7.05(2.46-21.9)$ & 0.0002 \\
\hline Model 2 & 1 & $1.95(0.73-5.42)$ & 0.18 & $2.17(0.73-6.67)$ & 0.16 & $8.20(2.51-29.2)$ & 0.0004 \\
\hline Model 3 & 1 & $2.32(0.84-6.86)$ & 0.11 & $2.39(0.78-7.65)$ & 0.13 & $9.40(2.73-35.8)$ & 0.0003 \\
\hline \multicolumn{8}{|c|}{ Cognitive deterioration: sex-specific serum UA quartiles } \\
\hline Single-variable analysis & 1 (reference) & $0.66(0.24-1.76)$ & 0.41 & $2.02(0.87-4.86)$ & 0.1 & $5.15(2.22-12.7)$ & 0.0001 \\
\hline \multicolumn{8}{|l|}{ Multivariate analyses } \\
\hline Model 1 & 1 & $0.53(0.18-1.46)$ & 0.22 & $2.10(0.86-5.37)$ & 0.1 & $4.04(1.64-10.5)$ & 0.0023 \\
\hline Model 2 & 1 & $0.55(0.19-1.55)$ & 0.26 & $2.49(0.96-6.73)$ & 0.060 & $5.46(1.88-16.9)$ & 0.0016 \\
\hline Model 3 & 1 & $0.58(0.19-1.70)$ & 0.32 & $2.83(1.05-8.09)$ & 0.040 & $6.04(1.99-19.8)$ & 0.0013 \\
\hline \multicolumn{8}{|c|}{ WML: non-sex-specific serum UA quartiles } \\
\hline Single-variable analysis & 1 (reference) & $0.31(0.04-1.39)$ & 0.13 & $2.06(0.69-6.59)$ & 0.2 & $0.92(0.25-3.22)$ & 0.89 \\
\hline \multicolumn{8}{|l|}{ Multivariate analyses } \\
\hline Model 1 & 1 & $0.34(0.05-1.63)$ & 0.19 & $2.00(0.57-7.36)$ & 0.28 & $1.12(0.23-5.23)$ & 0.88 \\
\hline Model 2 & 1 & $0.42(0.05-2.23)$ & 0.32 & $2.45(0.56-11.5)$ & 0.23 & $1.44(0.22-9.64)$ & 0.7 \\
\hline Model 3 & 1 & $0.43(0.06-2.29)$ & 0.33 & $2.46(0.55-11.9)$ & 0.24 & $1.57(0.22-11.4)$ & 0.65 \\
\hline \multicolumn{8}{|c|}{ WML: sex-specific serum UA quartiles } \\
\hline Single-variable analysis & 1 (reference) & $0.95(0.25-3.58)$ & 0.93 & $0.96(0.25-3.65)$ & 0.96 & $1.81(0.54-6.51)$ & 0.34 \\
\hline \multicolumn{8}{|l|}{ Multivariate analyses } \\
\hline Model 1 & 1 & $0.85(0.21-3.38)$ & 0.81 & $0.87(0.21-3.55)$ & 0.84 & $1.08(0.29-4.26)$ & 0.91 \\
\hline Model 2 & 1 & $0.93(0.22-4.02)$ & 0.92 & $1.11(0.23-5.25)$ & 0.89 & $0.97(0.20-4.85)$ & 0.97 \\
\hline Model 3 & 1 & $0.96(0.22-4.27)$ & 0.95 & $1.05(0.21-5.08)$ & 0.95 & $1.02(0.20-5.24)$ & 0.98 \\
\hline
\end{tabular}

All participants were divided into quartiles using common cutoff values (group 1 [ $n=61]:<4.7 \mathrm{mg} / \mathrm{dL}$, group 2 [ $n=63$ ]: $4.7-5.4 \mathrm{mg} / \mathrm{dL}$, group 3 [ $n=49]$ : 5.5-6.2 $\mathrm{mg} / \mathrm{dL}$, and group $4[n=55]:<6.2 \mathrm{mg} / \mathrm{dL}$ ). Male and female participants were divided into quartiles using different cutoff values, which were calculated separately by sex (group 1 [ $n=59$ ]: male $<5.5 \mathrm{mg} / \mathrm{dL}$ and female $<4.2 \mathrm{mg} / \mathrm{dL}$, group 2 [ $n=61]$ : male $5.5-6.2 \mathrm{mg} / \mathrm{dL}$ and female $4.2-4.8 \mathrm{mg} / \mathrm{dL}$, group 3 [ $n=60]$ : male: $6.3-6.9$ $\mathrm{mg} / \mathrm{dL}$ and female $4.9-5.4 \mathrm{mg} / \mathrm{dL}$, and group $4[n=48]$ : male $>6.9 \mathrm{mg} / \mathrm{dL}$ and female $>5.4 \mathrm{mg} / \mathrm{dL}$ ). In the single-variable analysis, the diagnosis of cognitive deterioration/normal was regressed only onto serum UA levels and crude ORs are presented. In multivariate analyzes, the following variables were added and multivariate-adjusted ORs are presented: model 1: age, sex, BMI, and education, model 2: variables in model 1 plus systolic blood pressure, fasting blood sugar, triglyceride, LDL-cholesterol, HDL-cholesterol levels, eGFR, and the presence of WML (for cognitive deterioration/non-sex-specific serum UA quartiles and cognitive deterioration/sex-specific serum UA quartiles only), model 3: variables in model 2 plus alcohol, smoking, and exercise. OR, odds ratio; WML, white matter lesions; CI, confidence interval; UA, uric acid; WML, white matter lesion.

The baseline characteristics of the 228 participants (105 male individuals, 123 female individuals; age range: $33-88$ years) are presented in Table 1 . Serum UA levels ranged from 3.2 to $10.1 \mathrm{mg} / \mathrm{dL}$ for men and from 2.4 to $7.9 \mathrm{mg} / \mathrm{dL}$ for women, and were significantly higher for men $\left(t_{226}=9.59, p<0.0001\right)$. Serum UA levels positively correlated with body weight ( $r=0.447, p<0.001)$, height $(r=0.342, p<0.0001)$, BMI $(r=0.320, p<0.0001)$, and inversely correlated with HDL-cholesterol levels $(\mathrm{r}=-0.289, p<0.0001)$ and eGFR $(r=-3974$, $p<0.0001)$. The participants with high levels of serum triglycerides showed significantly higher serum UA levels than those without $\left(t_{226}=-4.36, p<0.0001\right)$. Smoking $\left(t_{226}=-3.20\right.$, $p=0.0016)$ and alcohol $\left(t_{226}=3.27, p=0.0013\right)$ were the 2 habits found to be significantly associated with the elevation in serum UA levels.

To examine the association between serum UA levels and cognitive deterioration using logistic regression analyses, we divided the participants into quartiles based on their serum UA levels. The participants whose serum UA concentrations were under $4.7 \mathrm{mg} / \mathrm{dL}, 4.7-5.4$ $\mathrm{mg} / \mathrm{dL}, 5.5-6.2 \mathrm{mg} / \mathrm{dL}$, and over $6.2 \mathrm{mg} / \mathrm{dL}$ were classified into groups $1,2,3$, and 4, respectively. When diagnosis of cognitive deterioration/normal was regressed only on serum UA levels, the participants in the highest quartile of serum UA levels (group 4) had a significantly higher risk of cognitive deterioration compared to those in the lowest quartiles (group 1) $(\mathrm{OR}=5.99,95 \% \mathrm{CI}=2.56-15.2$; Table 2$)$. Individuals in groups 2 and 3 also showed a higher 
risk for cognitive deterioration; however, this did not attain statistical significance $(\mathrm{OR}=1.50$, $95 \% \mathrm{CI}=0.60-3.94 ; \mathrm{OR}=2.31,95 \% \mathrm{CI}=0.91-6.11$, respectively). We next confirmed this association using multivariate logistic regression models adjusted for potential confounders. In model 1, diagnosis of cognitive deterioration/normal was regressed on age, sex, BMI, education, and UA levels. In model 2, systolic blood pressure, fasting blood sugar, triglyceride, HDL-cholesterol, and LDL-cholesterol levels, alongside eGFR, and the presence of WML were also included. Finally, we added parameters related to lifestyle habits such as smoking, alcohol consumption, and regular exercise in model 3. A UA-dependent increase in the risk of cognitive deterioration was consistently observed in each regression model. The OR (95\% CI) of the highest quartile compared to the lowest quartile was 7.05 (2.46-21.9), 8.20 (2.51-29.2), and 9.40 (2.73-35.8) in models 1,2 , and 3, respectively.

As shown in Table 1, the average concentration of serum UA was higher in male than in female participants. Meanwhile, the cognitive deterioration group included more male than female participants, although the difference was not statistically significant. Therefore, to correct for possible overestimation of the risk of cognitive deterioration due to sex differences in serum UA levels, we subsequently divided the participants into quartiles according to serum UA cutoff values, which were calculated separately by sex. In the group of male participants, the cutoff values for groups $1,2,3$, and 4 were $<5.5,5.5-6.2,6.3-6.9$, and $>6.9 \mathrm{mg} / \mathrm{dL}$, respectively. In the group of female participants, the cutoff values for groups $1,2,3$, and 4 were $<4.2$, $4.2-4.8,4.9-5.4$, and $>5.4 \mathrm{mg} / \mathrm{dL}$, respectively. Following this, single-variable and multivariate logistic regression analyses were performed on these quartiles using the same set of covariates as in previous analyses (Table 2). This alternative analysis further confirmed the increased risk of cognitive deterioration in the highest quartile of serum UA levels. A single-variable analysis gave a crude OR of $5.15(95 \% \mathrm{CI}=2.22-12.7)$. In multivariate analyses, the adjusted OR (95\% CI) was 4.04 (1.64-10.5), 5.46 (1.88-16.9), and 6.04 (1.99-19.8) in models 1, 2, and 3 , respectively. Group 3 also showed a greater than twofold increase in the risk of cognitive deterioration in each model; however, statistical significance was reached only in model 3 (adjusted $\mathrm{OR}=2.67,95 \% \mathrm{CI}=1.01-7.51$ ). On the other hand, unlike the previous analysis, the risk for group 2 was estimated to be lower than that for group 1 . However, neither analysis yielded significant results for the relationship between groups 1 and 2 .

We also analyzed the association between serum UA levels and the presence of WML in order to examine whether cognitive deterioration in people with high UA levels is caused by ischemic changes in the brain. To achieve this, we performed a logistic regression analysis with the presence of WML as a dependent variable (Table 2). Independent variables, other than the presence of WML, were the same as those in the previous analyses. In non-sexspecific quartiles, an approximately twofold increase in OR was observed in single-variable and multivariate analyses of group 3; however, these were found not to be statistically significant. In addition, no such tendency was observed in sex-specific quartile analyses. None of these analyses could prove any significant association between serum UA levels and the presence of WML. Finally, we checked the association between the presence of WML and cognitive deterioration. Ten out of 64 cognitive deterioration cases and 12 out of 163 normal cases had WML. The $\chi^{2}$ test did not show any significant differences in the prevalence of WML between cognitive deterioration cases and normal cases $\left(\chi_{(2, n=228)}^{2}=3.585, p=0.058\right)$.

\section{Discussion}

In this study, we demonstrated that there is a positive association between concentrations of serum UA and cognitive deterioration in a Japanese population. The participants with serum UA levels in the highest quartile were estimated to have at least a fourfold 
higher risk of presenting with cognitive deterioration compared to those in the lowest quartile. This association remained statistically significant even after adjusting for confounding factors such as age, sex, BMI, education, systolic blood pressure, fasting blood sugar, triglyceride, HDL-cholesterol, LDL-cholesterol, and serum creatinine levels, as well as a smoking habit, alcohol consumption habit, and regular exercise. On the other hand, no positive association was observed between serum UA levels and the presence of WML. The same tendency was also observed in further sets of analyses in which the cutoff values of serum UA levels were determined separately by sex. This result further suggests that a UA-dependent increase in the risk of cognitive deterioration is common in both male and female individuals.

While there is a lot of controversy regarding the influence of UA on cognitive function, our results clearly support the idea that mildly elevated serum UA levels have a negative impact on cognitive function, which is sufficient to negate any potential benefits caused by the antioxidant properties of UA. Previous studies have mainly focused on UA as a risk factor for ischemic changes in the brain. An MRI-based quantitative study demonstrated that mildly elevated serum UA levels are associated with an increased burden of cerebral ischemic pathology, particularly in older adults [17]. Concentrations of UA in the cerebrospinal fluid were shown to be significantly higher in patients with vascular dementia but lower in patients with AD compared to healthy controls [18]. In this context, it is slightly unexpected that no significant association between serum UA levels and the presence of WML was observed in our population, although it should be recognized that there are limitations to using WML as an indicator of cerebral ischemia. One possible explanation for this discrepancy is that even subclinical levels of ischemic changes caused by UA can negatively impact cognitive function. Another argument is that there might be an unknown mechanism underlying the detrimental effects of elevated serum UA levels on cognitive function. More biological studies are needed to answer these questions.

Besides this study, at least 2 other cross-sectional studies have supported the hypothesis that increased serum UA levels are associated with cognitive deterioration $[10,11]$. On the other hand, there has been one prospective population-based cohort study showing that increased serum UA levels are associated with better cognitive function later in life [19]. There are 2 possible explanations for this discrepancy. First, this may result from variations in the causes of elevations in serum UA levels, which are generally classified into 3 types: increased production of UA, decreased excretion of UA, and mixed type. It might be expected that the frequency of each type varies depending on the target population considered, since serum UA concentrations are influenced by many factors such as nutrition, alcohol consumption, genetic factors, and renal function. Moreover, hyperuricemia in the Japanese population is characterized by a high prevalence of the decreased excretion type, with the mixed and increased production types being less frequent [20]. Variations in the causes of hyperuricemia may differentially affect cognitive function. Second, this discrepancy may mean that the short-term effects of UA on cognitive function are different from its long-term effects. Most of the studies that showed that UA had an adverse effect on cognitive function were cross-sectional studies, in which UA measurement and cognitive tests were performed around the same time $[10,11]$. On the other hand, the study showing a beneficial effect of UA focused on the cognitive outcomes of people with higher UA levels over the course of a decade [19]. Furthermore, progression of cognitive deterioration itself surely affects serum UA levels through changes in lifestyle, such as nutrition and exercise. From this perspective, the lower serum UA levels observed in patients with $\mathrm{AD}$ are not necessarily the cause of AD; however, they may instead reflect changes in lifestyle. To resolve this controversy, a more detailed time course analysis focusing on the association between cognitive function and serum UA levels is needed. 
Our present study had at least 2 limitations. First, the "cognitive deterioration" group defined in this study is likely heterogeneous in the clinical stages of dementia, and possibly included not only pre-MCI but also MCI and even mild dementia since participants were not clinically diagnosed by a physician and there was no age cutoff or cognitive cutoff for participation. Thus, information relating to clinical diagnosis cannot be obtained from this study. Another possibility is that other disorders that can affect cognition, such as depression, hypothyroidism, and renal dysfunction, were not excluded from this study. Second, since we drew the data from voluntary participants of a health checkup, the population we analyzed is prone to include people with a high interest in health issues. Thus, our target population may not precisely reflect the general Japanese population because of this possible selection bias. Further studies based on larger populations are required to solve this problem.

We conclude that elevated serum UA is an independent risk factor for cognitive deterioration in the Japanese population. Our results suggest the possibility that the vascular pathology caused by UA alone is not the cause of cognitive deterioration. To elucidate how serum UA affects/is affected by changes in cognitive deterioration, further detailed studies focusing on the time course of changes in serum UA levels and cognitive function are needed.

\section{Acknowledgements}

We thank Nagisa Miyamori, Miyuki Odawara, and Mayumi Namba for their support with the neuropsychological tests.

\section{Disclosure Statement}

None of the authors have any conflicts of interest to declare.

\section{References}

$>1$ Fang J, Alderman MH: Serum uric acid and cardiovascular mortality the NHANES I epidemiologic follow-up study, 1971-1992. National Health and Nutrition Examination Survey. JAMA 2000;283:2404-2410.

-2 Niskanen LK, Laaksonen DE, Nyyssonen K, Alfthan G, Lakka HM, Lakka TA, Salonen JT: Uric acid level as a risk factor for cardiovascular and all-cause mortality in middle-aged men: a prospective cohort study. Arch Intern Med 2004;164:1546-1551.

-3 Bos MJ, Koudstaal PJ, Hofman A, Witteman JC, Breteler MM: Uric acid is a risk factor for myocardial infarction and stroke: the Rotterdam study. Stroke 2006;37:1503-1507.

-4 Rao GN, Corson MA, Berk BC: Uric acid stimulates vascular smooth muscle cell proliferation by increasing platelet-derived growth factor A-chain expression. J Biol Chem 1991;266:8604-8608.

5 Kanellis J, Watanabe S, Li JH, Kang DH, Li P, Nakagawa T, Wamsley A, Sheikh-Hamad D, Lan HY, Feng L, Johnson RJ: Uric acid stimulates monocyte chemoattractant protein-1 production in vascular smooth muscle cells via mitogen-activated protein kinase and cyclooxygenase-2. Hypertension 2003;41:1287-1293.

6 Corry DB, Eslami P, Yamamoto K, Nyby MD, Makino H, Tuck ML: Uric acid stimulates vascular smooth muscle cell proliferation and oxidative stress via the vascular renin-angiotensin system. J Hypertens 2008;26:269275.

7 Khosla UM, Zharikov S, Finch JL, Nakagawa T, Roncal C, Mu W, Krotova K, Block ER, Prabhakar S, Johnson RJ: Hyperuricemia induces endothelial dysfunction. Kidney Int 2005;67:1739-1742.

-8 Leyva F, Anker SD, Godsland IF, Teixeira M, Hellewell PG, Kox WJ, Poole-Wilson PA, Coats AJ: Uric acid in chronic heart failure: a marker of chronic inflammation. Eur Heart J 1998;19:1814-1822.

-9 Ruggiero C, Cherubini A, Ble A, Bos AJ, Maggio M, Dixit VD, Lauretani F, Bandinelli S, Senin U, Ferrucci L: Uric acid and inflammatory markers. Eur Heart J 2006;27:1174-1181.

10 Schretlen DJ, Inscore AB, Jinnah HA, Rao V, Gordon B, Pearlson GD: Serum uric acid and cognitive function in community-dwelling older adults. Neuropsychology 2007;21:136-140. 
11 Ruggiero C, Cherubini A, Lauretani F, Bandinelli S, Maggio M, Di Iorio A, Zuliani G, Dragonas C, Senin U, Ferrucci L: Uric acid and dementia in community-dwelling older persons. Dement Geriatr Cogn Disord 2009;27:382389.

12 de Lau LM, Koudstaal PJ, Hofman A, Breteler MM: Serum uric acid levels and the risk of Parkinson disease. Ann Neurol 2005;58:797-800.

13 Rinaldi P, Polidori MC, Metastasio A, Mariani E, Mattioli P, Cherubini A, Catani M, Cecchetti R, Senin U, Mecocci P: Plasma antioxidants are similarly depleted in mild cognitive impairment and in Alzheimer's disease. Neurobiol Aging 2003;24:915-919.

14 Kim TS, Pae CU, Yoon SJ, Jang WY, Lee NJ, Kim JJ, Lee SJ, Lee C, Paik IH, Lee CU: Decreased plasma antioxidants in patients with Alzheimer's disease. Int J Geriatr Psychiatry 2006;21:344-348.

15 Fazekas F, Kleinert R, Offenbacher H, Payer F, Schmidt R, Kleinert G, Radner H, Lechner H: The morphologic correlate of incidental punctate white matter hyperintensities on MR images. AJNR Am J Neuroradiol 1991;12: 915-921.

-16 Matsuo S, Imai E, Horio M, Yasuda Y, Tomita K, Nitta K, Yamagata K, Tomino Y, Yokoyama H, Hishida A: Revised equations for estimated GFR from serum creatinine in Japan. Am J Kidney Dis 2009;53:982-992.

-17 Schretlen DJ, Inscore AB, Vannorsdall TD, Kraut M, Pearlson GD, Gordon B, Jinnah HA: Serum uric acid and brain ischemia in normal elderly adults. Neurology 2007;69:1418-1423.

18 Tohgi H, Abe T, Takahashi S, Kikuchi T: The urate and xanthine concentrations in the cerebrospinal fluid in patients with vascular dementia of the Binswanger type, Alzheimer type dementia, and Parkinson's disease. J Neural Transm Park Dis Dement Sect 1993;6:119-126.

19 Euser SM, Hofman A, Westendorp RG, Breteler MM: Serum uric acid and cognitive function and dementia. Brain 2009;132:377-382.

20 Yamanaka H, Kamatani N: Gout. Igaku to Yakugaku 1993;29:1357-1363. 